\title{
Competition and synergy patterns in nonhomogeneous ant-colony lattices.
}

\author{
J. C. Becceneri ${ }^{a 1}$, R. Sautter ${ }^{b}$, N. L. Vijaykumar ${ }^{a}$ and C.P. Camilo ${ }^{c}$. \\ ${ }^{a}$ Laboratory for Computing and Applied Mathematics (LAC), \\ National Institute for Space Research (INPE),C.P. 515, S. J. dos Campos - SP, 12001-970, Brazil. \\ ${ }^{b}$ CAP graduate program, INPE, S. J. dos Campos - SP, 12228-900, Brazil. \\ ${ }^{c}$ Universidade Cruzeiro do Sul (UNICSUL), São Paulo, Brazil.
}

Received on July 21, 2015 / accepted on 20 December, 2015

\begin{abstract}
In this paper we introduce, for the first time, an Ant Colony System (ACS) composed of two different populations of agents. The first population has more agents than the second while the second has more energy than the first. The agents are distributed in a square lattice according to their corresponding population. In our first approach of this nonhomogeneous system the formation and evolution of competition and synergy patterns are described by means of the Gradient Pattern Analysis (GPA) technique. Based on several preliminary results we discuss a new game prototype whose laws of success and convergence are given by means of symmetry breaking patterns in a nonhomogeneous $(4 \times 4)$ lattice.
\end{abstract}

Keywords: Intelligent agents, ant colony system, gradient pattern analysis, game theory, comp. mathematics.

\section{Introduction}

Meta-heuristic algorithms, such as Genetic Algorithms, Simulated Annealing, and Tabu search, guide the application and use of local improvement heuristics. Usually, a meta-heuristic is formally defined as an interactive generation process which guides a subordinate heuristic by combing intelligently different concepts for exploring and exploiting the search space, learning strategies that are used to structure information in order to find efficiently near-optimal solutions [7]. Over the past few years, they have become more and more important in applications to solve a wide range of problems [6].

The basic Ant System (AS) is based on the known behaviour of a group of ants trying to find food [3]. Recently, some modifications were proposed on the AS in order to improve the performance of the system in the solution of large size problems [4, 1]. They introduced a new transition rule, a different way of depositing pheromone, restrictions about the use of the tabu table and the use of local updates of pheromone trail to favor exploration. This new approach was called Ant Colony System (ACS).

This paper introduces an ACS composed of sub-colonies whose agents are distributed in a matricial structure working as a square graph. Figure 1 shows the lattice configuration for the simplest canonical prototype composed of two sub-colonies. This lattice is a 44 square matrix superposed by a 16-nodes graph, where the four internal nodes $(\mathrm{N})$ constitute the nucleus, and the twelve external nodes $(\mathrm{B})$ constitute the boundary. Note that, each sub-colony can be characterized by means of its initial position on the graph and by its initial amount of energy. For the nucleous the individual energies are equal to $1 / 4$ while for

\footnotetext{
${ }^{1}$ E-mail Corresponding Author: becce@lac.inpe.br
} 
M1: Initial Condition

$\begin{array}{llll}0.083 & 0.083 & 0.083 & 0.083\end{array}$

$\begin{array}{llll}0.083 & 0.250 & 0.250 & 0.083\end{array}$

$\begin{array}{llll}0.083 & 0.250 & 0.250 & 0.083\end{array}$

$\begin{array}{llll}0.083 & 0.083 & 0.083 & 0.083\end{array}$

\section{M3: Target 2}

$\begin{array}{lll}0.000 & 0.083 & 0.083\end{array}$

$\begin{array}{lll}0.083 & 0.250 & 0.250\end{array}$

$\begin{array}{lll}0.083 & 0.250 & 0.250\end{array}$

$\begin{array}{ll}0.083 & 0.083\end{array}$

$0.083 \quad 0.083$

\section{M2: Target1}

$\begin{array}{llll}0.083 & 0.083 & 0.083 & 0.083\end{array}$

$\begin{array}{llll}0.083 & 0.083 & 0.083 & 0.083\end{array}$

$\begin{array}{llll}0.083 & 0.083 & 0.083 & 0.083\end{array}$

$\begin{array}{llll}0.083 & 0.083 & 0.083 & 0.083\end{array}$

Figure 2 - Four examples of typical matrices involved in our simulations.

the boundary the individual energies are equal to $1 / 12$, so that the total energy of the system tends to 2. An interesting aspect of this geometrical configuration is that the dynamical states of the system can also be characterized by means of the instantaneous matricial patterns obtained during the system evolution.

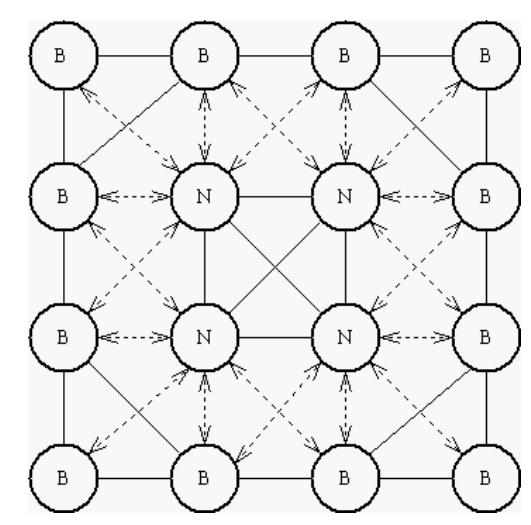

Figure 1 -The simplest canonical matrix-graph configuration composed of two sub-colonies $\mathrm{N}$ and $\mathrm{B}$.

Figure 2 shows four examples of typical matrices involved in our simulations: M1 is the canonical initial condition given by the criterion explained before; M2 is a target matrix representing a score in favor of the colony B and M3 is a target matrix representing a score in favor of the colony N; M4 is a typical attractor pattern that will be discussed later in Section 3.

A proper way to characterize the patterns is by means of the gradient pattern analysis (GPA) introduced by Rosa et al. [9, 10]. For applications, see also [11, 12, 8, 5, 13]. The spatial distribution of vectors in the gradient field $\nabla M$ will contain many vectors with the same magnitude, within a small tolerance, and these form symmetric pairs if they have opposite orientations, and asymmetric pairs otherwise. After removing all symmetric pairs of vectors, the number of asymmetric vectors is denoted by L. Any symmetry breaking can be quantified by connecting the middle points of the $\mathrm{L}$ vectors with I straight lines, generating a Delaunay triangulation $T_{D}(I, L)$.

The triangulation field is a fractional field whose dimension is less than two - the lattice dimension. A measure of the asymmetry is the difference $I-L$ normalized by $L$, the so-called Gradient Asymmetry $G_{a}=(I-L) / L$ where $I>L>0$. For symmetric patterns $L=0$ and by definition, $G_{a}=0$.

The $G_{a}$ parameter quantifies the degree of vectorial asymmetry and it is proportional to the symmetry breaking during the evolution of the gradient field pattern $P_{i}$.

For M1 the asymmetric gradient $\mathrm{Ga}$ is null, for the targets M2, M3 and M4 the values are 0.875, 1.125 and 1.563, respectively. The output from application of GPA on the matrixes M2 e M4 is shown in Figure 3. 

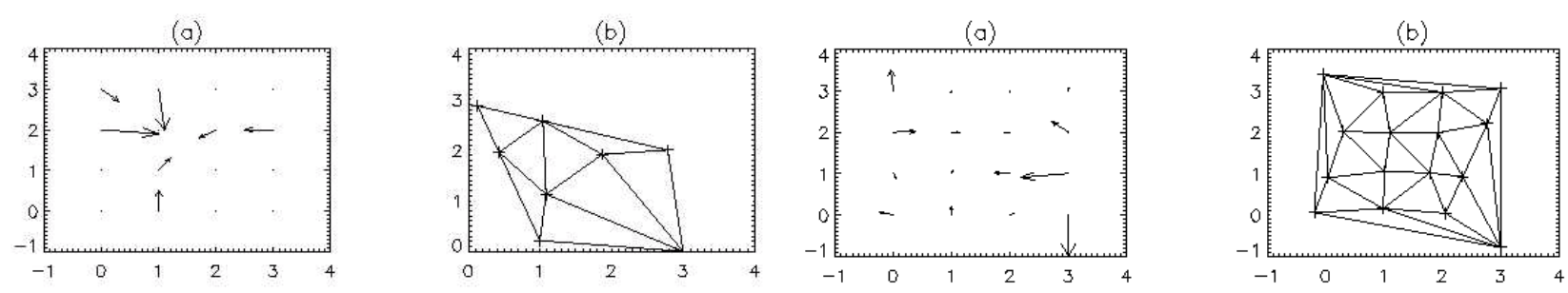

Figure 3 -The gradient pattern analysis output for matrices M2 (up) and M4 (down): (a) the asymmetric gradient field and (b) its respective triangulation field.

In the next section we give a complete description of our algorithm and its implementation. The results and interpretation are introduced in Section 3 and the concluding remarks in Section 4.

\section{The Algorithm Implementation}

Usually, a graph model is used to represent the dynamics of an ACS algorithm, where the ants move along the nodes of this graph. A node is chosen to be visited based on a probability function. The following probability function gives the best node to be visited in that moment:

$$
p_{i j}^{k}(t)=\frac{\left[\tau_{i j}(t)\right]^{\alpha}+\left[\eta_{i j}\right]^{\beta}}{\sum_{I \in J_{k}^{i}}\left[\tau_{i j}(t)\right]^{\alpha}+\left[\eta_{i j}\right]^{\beta}}
$$

Where $\tau_{i j}$ is the quantity of pheromone between $\mathrm{i}$ and $\mathrm{j}$, and $\eta_{i j}$ is the visibility that an ant stopped at node $\mathrm{i}$ has of node $\mathrm{j}$. The parameters $\alpha$ and $\beta$ control the relative weight of $\tau_{i j}(t)$ and $\eta_{i j}(t)$. The parameter $J_{i}^{k}$ inform the set of nodes that an ant $\mathrm{k}$, stopped at node $\mathrm{i}$, can visit.

As given by the ACS formalism the transition rules are characterized by the parameter $q_{0}$. Now, before going to a new node, we sample a new number, randomly, between 0 and 1 , and apply the following transition rule:

$$
J= \begin{cases}J_{i}^{k} & \text { if } q>q_{0} \\ \arg \max _{u \in J_{k}^{i}}\left\{\left[\tau_{i j}(t)\right]^{\alpha},\left[\eta_{i j}\right]^{\beta}\right\} & \text { if } q \leq q_{0}\end{cases}
$$

If $q<q_{0}$ then the best edge is chosen (exploitation), otherwise an edge is chosen according to Eq.(1) (biased exploration).

For the problem studied here, the visibility is a function of the established rules to move between the cells. Actually, the visibility is the inverse of the cost to extract some energy and depends on the main parameters of our algorithm that are:

- $\alpha$ and $\beta$ control the relative weight of $\tau_{i j}(t)$ and $\eta_{i j}(t)$;

- nit the number of iterations performed by the algorithm;

- seed is used to generate the random numbers.

For all test cases, the same relative weight for $\alpha$ and $\beta$ was considered. The target patterns were characterized by means of $G_{a}$ parameter. We found a discrete domain composed of twelve target values: four values for sub-colony $\mathrm{N}$ score and eight values for sub-colony $\mathrm{B}$ score. The follow table shows the values used in our prototype implementation.

The seed to generate the random numbers is entered at each execution of the program through the keyboard.

To work out the probability function, expressed by equation (1), it is necessary to define a function to express the desirability among the nodes and a function to express the pheromone in the arcs of the graph as shown in Figure 1. Next, these functions are justified. 
Let $\eta$ be the desirability matrix. In the Salesman Problem, where the nodes represent cities, the desirability is expressed by the inverse of the distance between two cities. Here the desirability is function of the established rules to realize the movement between the cells. These rules are represented in the diagram of Figure 4.

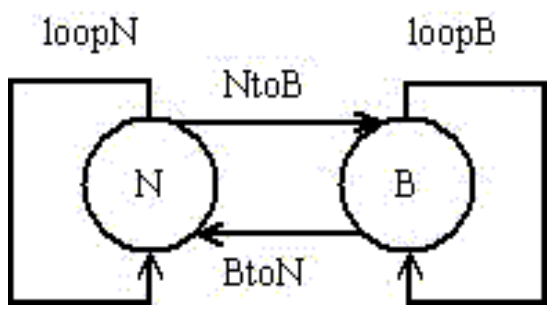

Figure 4 - Transition parameters.

An agent of type $\mathrm{N}$ has a desire to stay in itself expressed by the parameter loopN and to go to a $\mathrm{B}$ node expressed by NtoB. It cannot go to a node of the same type. The parameters loopB and BtoN apply to B nodes. If fair rules are desired, the following can be considered: the sum of all NtoB+loopN is equal to BtoN+loopB. This means that, for the initial condition, there is a situation of balance.

In this system it is said that a movement occurred when an agent performed a loop or moved to another node. When an agent of type B, for example, goes to a cell occupied by an agent of type $\mathrm{N}$, it is said that an invasion occurred (the cell occupied by $\mathrm{N}$ was invaded by $\mathrm{B}$ ). The same definition can be applied to define an invasion of $\mathrm{N}$ into $\mathrm{B}$.

In the case being studied, there are 4 agents of type $\mathrm{N}$ and 12 agents of type $\mathrm{B}$, so:

$$
\sum_{1}^{4}(N t o B+\operatorname{loop} N)=\sum_{1}^{12}(B t o N+\operatorname{loop} B)
$$

To test the ideas presented here, several test cases were performed, and, arbitrarily, we call a complete match a set of six cases. The values considered for a match example (composed of cases A to F) are shown in Table 2.

The matrix $\tau$ expresses the quantity of pheromone in the arcs, $\tau$ ij represents the quantity of pheromone between the arc $\mathrm{j}$ and $\mathrm{i}$ and $\rho$ is the decay parameter. The covered path receives an increment of $\Delta$.

\begin{tabular}{|c|c|c|c|c|c|c|c|}
\hline Case & $\tau_{0}$ & $\rho$ & $\Delta$ & NtoB & loopN & BtoN & loopB \\
\hline A & 1.00 & 0.10 & 1.50 & 2.70 & 0.30 & 0.90 & 0.10 \\
B & 1.00 & 0.10 & 1.50 & 0.90 & 0.10 & 0.10 & 0.90 \\
C & 1.00 & 0.10 & 1.50 & 0.75 & 0.25 & 0.25 & 0.75 \\
D & 1.00 & 0.90 & 1.50 & 0.75 & 0.25 & 0.25 & 0.75 \\
E & 1.00 & 0.20 & 1.50 & 2.70 & 0.30 & 0.90 & 0.10 \\
F & 1.00 & 0.80 & 1.50 & 2.70 & 0.30 & 0.90 & 0.10 \\
\hline
\end{tabular}

The initial quantity of this substance is equal for all arcs $\tau_{0}$. After each iteration, the quantity of pheromone in each arc is updated and if the arc $(a, b)$ represents the path between the agents involved in the iteration, then the following equations are applied as rules to update the pheromone:

$$
\begin{aligned}
\tau[a][b] & =\tau[a][b] \Delta \\
\tau[i][j] & =\tau[i][j] \rho
\end{aligned}
$$

Now, the last parameter used to implement this model is explained. To each type of agent a value is associated and named significance level. This value can be considered as the initial energy of that agent. Each type of agent has a lower bound and an upper bound for its significance level, which define an interval of variation for the values that can be assumed by the significance level. 
$E_{0} B$ is the initial value for the significance level of $\mathrm{B}, \mathrm{LBB}$ is the lower bound for $\mathrm{B}$, UBB is the upper bound for $\mathrm{B}$ and $\mathrm{EB}$ is the current value. $E_{0} N, \mathrm{LBN}, \mathrm{UBN}$ and $\mathrm{EN}$ are the parameters for N. Figure 5 shows the logic applied to work out the new significance level of invaded cell is explained. Consider that an agent of type $\mathrm{B}$ is invading a cell occupied by an agent of type $\mathrm{N}$ and that $\mathrm{x}$ is an auxiliary variable.

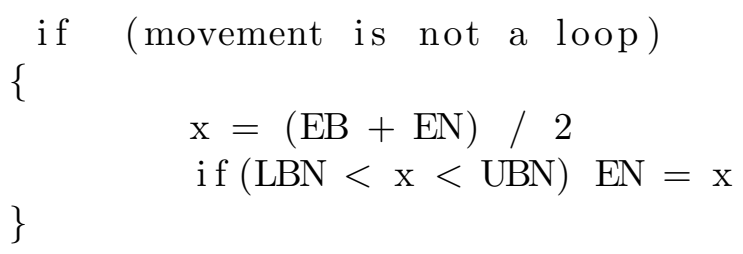

Some considerations are necessaries:

- If the movement is a loop, nothing is done;

- If $\mathrm{x}$ is not between LBN and UBN, the significance level of $\mathrm{N}$ is not altered;

- The rule to calculate $\mathrm{x}$ can be defined in different ways. Note that, the average between EB and EN is a simple way to define new levels.

The values considered, for the test cases $\mathrm{A}$ to $\mathrm{F}$ are shown in Table 3. To understand the pseudo-code generated consider nag the number of different agents and at represents the agent type (B or $\mathrm{N})$, and a pseudo-code can be expressed as follows:

\begin{tabular}{|c|c|c|c|c|c|}
\hline$E_{0} B$ & LBB & UBB & $E_{0} N$ & LBN & UBN \\
\hline $1 / 12$ & 0 & $(1 / 12)+(1 / 4)$ & $1 / 4$ & 0 & $(1 / 12)+(1 / 4)$ \\
\hline
\end{tabular}

Table 3 - Values to calculate the significant level of the agents.

Results were plotted where simulations based on certain features corresponded to the calculated Ga targets. This is shown in Figure 6, by means of the plots of $G_{a}$ versus Frame-Steps. Solid lines correspond to Boundary sub-colonies targets whereas dotted lines correspond to Nucleus sub-colonies target. The match result is characterized by the total scores attained during the six evolutions. One more interesting study can be added in which $G_{a}$ values move to a convergence. At the point where a convergence starts one can mark whether the point of convergence is close to a sub-colony target. This feature can be named as attractors.In the case of the example studied for this paper, only the Boundary sub-colony has been privileged with one attractor characterized by means of convergence after step 110 for case B (asterisc mark ).

\section{Concluding Remarks}

We study a new kind of ACS by running experiments to understand its operability as an extended game prototype. The results show that this nonhomogeneous ant colony system, composed of two different subcolonies, outperforms cooperative and competitive evolutionary computation whose dynamical characteristics in space and time can be characterized by the geometrical patterns of the corresponding graph gradient fields. Generalization of this hybrid extended ACS system can be easily done for matrix-graph of size NxN and in such cases, the characterization of symmetry breaking in the gradient field of a given nonhomogeneous ACS can be a useful tool to understand complex dynamical regimes as spatio-temporal intermittency, selforganization and turbulence in the context of the game theory. We believe that this approach can get closer to the problems of non-linear physics and meta-heuristic computation for further applications on scaling and chaotic physics, biophysics, economics and risk management theory.

Acknowledgments: This work was supported by CNPq-Brazil. The authors thank R.R.Rosa for discussion on GPA and for his original idea on the ant-colony lattice model introduced in this paper. 


\section{References}

[1] Banabeau, E., Social insect colonies as complex adaptative systems, Ecosystems 1: 437-443, 1998. doi: $10.1007 / \mathrm{s} 100219900038$

[2] Banabeau, E., Dorigo, M., Theraulaz, G. Inspiration for optimization from social insect behaviour, Nature 406: 39-42, 2000. doi:10.1038/35017500

[3] Dorigo, M. Optimization, learning and natural algorithms. Ph.D. Thesis, Politecnico di Milano, Italy, 1992.

[4] Dorigo, M., Gambardella, L.M. Ant colonies for the travelling salesman problem, Biosystems 43: 73-81, 1997.

[5] da Silva, A.F., Rosa, R.R., Roman, L.S., Veje, E., Pepe, I. Characterization of asymmetric fragmentation patterns in SFM images of porous silicon, Solid State Commun 113: 703-708, 2000.

[6] Jones, D.F., Mirrazavi, S.K., Tamiz, M., Multi-objective meta-heuristics: An overview of the current state-of-the-art, European Journal of Operational Research 1: 1-9, 2002.

[7] Osman, I. H., Lapporte, G. Metaheuristics: a bibliography, Annals of Operations Research 63: 513-623, 2014.

[8] Ramos, F., Rosa, R.R., Rodrigues Neto, C., Zanandrea, A., Generalized complex entropic form for gradient pattern analysis of spatio-temporal dynamics, Physica A 283: 171-174, 2000.

[9] Rosa, R.R., Sharma, A.S., Valdivia, J.A. Characterization of localized turbulence in plasma extended systems, Physica A 257: 509-514, 1998.

[10] Rosa, R.R., Sharma, A.S., Valdivia, J.A. Characterization of asymmetric fragmentation patterns in spatially extended systems, International Journal of Modern Physics C 10:147-163, 1999.

[11] Rosa, R.R., Pontes, J., Christov, C.I., Ramos, F.M., Neto, C.R., Rempel, E.L., Walgraef, D. Gradient pattern analysis of Swift-Hohenberg dynamics: phase disorder characterization, Physica A 283: 156-159, 2000 .

[12] Rosa, R.R., Sawant, H.S., Cecatto, J.R., Neto, C.R., Lopes, V.C.A., Subramanian, K.R., Fernandes, F.C.R., Saito, J.H., Moron, C.E., Mucheroni, M.L., Furuya, N., Mascarenhas, N., Phenomenological dynamics of coronal loops using a neural network approach, Advances in Space Research 25: 1917-1921, 2000 .

[13] Neto, C.R., Rosa, R.R., Ramos, F.M. Pattern characterization: A case study in osmosedimentation, International Journal of Modern Physics C 12: 1261-1269, 2001. 